\title{
Estado nutricional e insegurança alimentar de adolescentes e adultos em duas localidades de baixo índice de desenvolvimento humano
}

\author{
Nutritional status and food insecurity of \\ adolescents and adults in two cities \\ with a low human development index
}

Juliana Souza OLIVEIRA' ${ }^{1}$

Pedro Israel Cabral de LIRA ${ }^{2}$

Ida Cristina Leite VERAS ${ }^{3}$ in memorian

Sandra Regina MAIA²

Maria da Conceição Chaves de LEMOS 4

Sonia Lucia Lucena Sousa de ANDRADE²

Maurício José VIANA JUNIOR ${ }^{5}$

Fernanda Cristina de Lima PINTO6

Vanessa Sá LEAL ${ }^{6}$

Malaquias BATISTA FILHO’

\section{RE S U M O}

\section{Objetivo}

Descrever a situação da (in)segurança alimentar e sua relação com o estado nutricional de adolescentes e adultos em duas localidades, Gameleira, zona da mata de Pernambuco e São João do Tigre, zona semi-árida da Paraíba, ambas caracterizadas pelo baixo índice de desenvolvimento humano.

\footnotetext{
$\overline{1}$ Universidade Federal de Alagoas, Faculdade de Nutrição. Campus A.C. Simões, Av. Lourival Melo Mota, s/n., Tabuleiro dos Martins, 57072-970, Maceió, AL, Brasil. Correspondência para/Correspondence to: J.S. OlIVEIRA. E-mails: <juliana_nutricao@yahoo.com.br>; <juliana_nutricao@oi.com.br>.

2 Universidade Federal de Pernambuco, Departamento de Nutrição. Recife, PE, Brasil.

${ }^{3}$ Hospital da Restauração de Pernambuco. Recife, PE, Brasil.

${ }^{4}$ Hospital Agamenon Magalhães, Unidade de Diabetes e Endocrinologia. Recife, PE, Brasil.

5 Bolsista do Programa Institucional de Bolsas de Iniciação Científica, Universidade de Pernambuco, Instituto Materno Infantil Prof. Fernando Figueiredo, Curso de Medicina. Recife, PE, Brasil.

${ }^{6}$ Nutricionistas. Recife, PE, Brasil.

7 Universidade Federal de Pernambuco, Programa de Pós-Graduação em Nutrição. Recife, PE, Brasil.
} 
454 | J.S. OLIVEIRA et al.

\section{Métodos}

Estudo transversal, com 501 famílias em Gameleira (PE) e 458 famílias, em São João do Tigre (PB), com 1528 e 1163 adolescentes e adultos, respectivamente. A (in)segurança alimentar foi determinada pelo questionário de Cornell (Estados Unidos da América), ajustado para realidade nacional (Escala Brasileira de Insegurança Alimentar), enquanto o estado nutricional foi estabelecido pelo Índice de Massa Corporal.

\section{Resultados}

A insegurança alimentar foi caracterizada em quase 90,0\% das famílias dos dois municípios, predominando as condições de insegurança moderada (40,2\%) em São João do Tigre (PB) e grave (36,9\%) em Gameleira (PE). Nas duas localidades, a insegurança alimentar prevaleceu nas famílias com adolescentes. O baixo peso para altura (proxi de desnutrição) apresentou proporções muito baixas em todos os grupos etários, enquanto a condição sobrepeso/obesidade prevaleceu entre os adultos, chegando a alcançar $58,6 \%$ das observações ( $\geq 40$ anos, em São João do Tigre). Não foi encontrada associação estatística entre a insegurança alimentar e suas formas moderada e grave e a desnutrição.

\section{Conclusão}

A escala brasileira de avaliação da (in)segurança alimentar se comporta mais como um indicador psicossocial de vulnerabilidade, não apresentando a esperada consistência com a proporção da desnutrição de adolescentes e adultos identificada por este estudo, sob o aspecto antropométrico, mesmo em populações de pobreza praticamente generalizada.

Termos de indexação: Adultos. Estado nutricional. Estudos transversais. Saúde do adolescente. Segurança alimentar e nutricional.

\section{A B S T R A C T}

\section{Objective}

The objective of this study was to describe the food insecurity profile and its relationship with the nutritional status of adolescents and adults of two cities in Northeast Brazil: (Gameleira (PE), located in the sugarcane area of Pernambuco and São João do Tigre (PB), located in the semiarid area of Paraíba), both characterized by a low human development index.

\section{Methods}

This is a cross-sectional study with 501 families from Gameleira (PE) and 458 families from São João do Tigre (PB), totaling 1528 adolescents and 1163 adults. Food insecurity was determined by Cornell's questionnaire (United States of America), localized for Brazil (Brazilian Food Insecurity Scale) while the nutritional status was determined by calculating the body mass index.

\section{Results}

Almost $90 \%$ of the families of both cities were on food insecurity. Moderate food insecurity was more common in São João do Tigre (PB) (40.2\%) and severe food insecurity in Gameleira (PE) (36.9\%). In the two cities, food insecurity prevailed in families with adolescents. There was a low prevalence of low weight-forheight (proxy for malnutrition) in all age groups, and a high prevalence (58.6\%) of overweight and obesity in adults 40 years or older in São João do Tigre. There was no statistical association between moderate and severe food insecurity and malnutrition.

\section{Conclusion}

The Brazilian scale to assess food insecurity is more of a psychosocial indicator of vulnerability. Under the anthropometric standpoint, the malnutrition rates found by this study among adolescents and adults were not consistent with their food insecurity statuses, even though poverty in these populations was widespread.

Indexing terms: Adults. Nutritional status. Cross-sectional studies. Adolescent health. Food security.

\section{N T R O D U ÇÃ O}

Ao assumir diferentes configurações em seu trânsito histórico nos últimos 60 anos, a
Segurança Alimentar e Nutricional (SAN) pode e deve ser compreendida, em sua conceituação mais atual, como a condição (de fato, um direito humano) que assegura a todas as pessoas o acesso 
físico, econômico e cultural a um elenco básico de alimentos capaz de suprir, de forma regular e permanente, a cobertura das necessidades biológicas de energia e nutrientes. Esta condição deve ser assegurada em um contexto de pleno atendimento a outros direitos fundamentais, como a educação, a moradia, a saúde e o bem estar social, dentro de um processo autônomo e sustentável de desenvolvimento, tendo como suporte o exercício de uma atividade laboral ética e culturalmente aceitável ${ }^{1,2}$. Embora alguns objetivos da SAN possam parecer ainda utópicos, na realidade, já constituem uma carta de princípios políticos, éticos e programáticos, referenciados por quase todos os países.

Nessa perspectiva, a SAN se colocou como consenso na Segunda Conferência Mundial de Alimentos ${ }^{3}$, na Conferência de Atenção Primária de Saúde, na Rússia ${ }^{4}$, nos compromissos da Reunião de Cúpula das Nações Unidas ${ }^{5}$ e na Cúpula Mundial de Alimentação ${ }^{6}$. Na Reunião de Nova York, se explicitou que a segurança alimentar domiciliar, além de um objetivo em si, constituía um pré-requisito para prevenir deficiências nutricionais, como o baixo peso ao nascer, a desnutrição energético-protéica (DEP), a hipovitaminose $A$, as anemias e a deficiência de iodo. Mais modernamente, com a valorização epidemiológica das doenças crônicas não transmissíveis, o conceito e a prática da SAN passam a se estender ao domínio da alimentação e dos estilos de vida saudáveis, ultrapassando, portanto, o marco dos processos carenciais da nutrição $0^{7,8}$.

Na medida em que representa um conceito novo e em rápido processo de mudança, a SAN necessita de um ou vários instrumentos que possibilitem avaliar sua própria condição, em termos populacionais, em grupos específicos e, finalmente, em instância familiar e até individual. Nesse sentido, na década de 1980, pesquisadores da Universidade de Cornell, desenvolveram um protótipo de avaliação qualitativa (a chamada escala de Cornell) composta por 10 perguntas, compreendendo simultaneamente a percepção da preocupação dos problemas relativos à quantidade de alimentos e à qualidade da dieta ${ }^{9-12}$. Na década de 90 , com base nesta escala e em outras similares, como a do Community Childhood Hunger Identification Project (CCHIP), estudiosos do Departamento de Agricultura dos Estados Unidos aperfeiçoaram o método, que passou a incluir 15 quesitos e 3 subitens. Com a nova configuração, desde 1995, o método passou a ser aplicado pelo Departamento do Censo e nas pesquisas periódicas de saúde e nutrição dos Estados Unidos ${ }^{1,9}$.

Para sua validação no Brasil, foram realizados oito grupos focais, entre 2002 e 2004, em quatro municípios brasileiros (Campinas, João Pessoa, Manaus e Goiânia), mediante estudo multicêntrico apoiado pela Organização Pan-Americana de Saúde, resultando na elaboração e na validação da Escala Brasileira de Insegurança Alimentar (EBIA $)^{12-14}$.

Com a nova formatação, esta escala foi utilizada pelo Instituto Brasileiro de Geografia e Estatística (IBGE) como tema especial da Pesquisa Nacional por Amostra de Domicílios (PNAD) ${ }^{12}$, sendo pertinente considerar que o Brasil é o país que reúne a experiência mais extensa do mundo em termos do uso massivo deste instrumento.

Como resultado desta aplicação extensiva à realidade brasileira, demonstrou-se que 39,8\% da população encontravam-se em situação de insegurança alimentar (IA), sendo 18,0\% leve, $14,1 \%$ moderada e $7,7 \%$ grave. No Nordeste, a condição de insegurança alcançou 59\% das famílias, chegando a 52,1\% na região Norte ${ }^{12}$. Desde que, em última instância, um dos objetivos da segurança alimentar deve ser o alcance de uma boa situação nutricional, um dos aspectos conceitualmente conflitivos destes resultados seria a marcante discordância com o estado nutricional da população brasileira em anos recentes, e, mais especificamente, com o quadro epidemiológico prevalente no Nordeste Brasileiro ${ }^{15-17}$. Ressalta-se, em princípio, a grande discordância entre as elevadas freqüências da insegurança alimentar, nacional e regional, em contraste com baixas prevalências de desnutrição em crianças, mesmo 
nos espaços geográficos mais pobres, como o Nordeste e o Norte do Brasil.

Nessas circunstâncias, considerou-se oportuno analisar a relação entre os níveis de (in)segurança alimentar e o estado nutricional de adolescentes e adultos em duas áreas de reconhecida vulnerabilidade socioeconômica, em uma conjuntura de rápida transição epidemiológica e nutricional.

O município da Gameleira localiza-se na Mata Meridional de Pernambuco, em um espaço geográfico típico da tradicional economia açucareira: monocultura, com elevada concentração de renda, mercado de trabalho instável entre a estação de máximo emprego (setembro/março) e o período de entressafra (abril/agosto), quando a ocupação do trabalho braçal cai para níveis mínimos na cidade e no campo, estabelecendo-se um período crítico de trabalhos avulsos, subemprego ou completo desemprego.

No ano 2000, segundo o Censo Demográfico do IBGE, o município possuía uma população de 25200 habitantes, dos quais 69,4\% residentes na área urbana e 30,6\% no meio rural. Mesmo no espaço urbano, as atividades econômicas acham-se estreitamente dependentes dos efeitos e das demandas da agroindústria canavieira. Segundo o Programa das Nações Unidas para o Desenvolvimento (PNUD, 2006), o Índice de Desenvolvimento Humano (IDH) do Município era de 0,590 , representado pelos sub-índices educação $(0,648)$, longevidade $(0,627)$ e renda $(0,496)$, com $73,6 \%$ das famílias vivendo em condição de pobreza absoluta ${ }^{18,19}$.

São João do Tigre situa-se na micro-região dos Cariris Velhos, no Estado da Paraíba, compondo um conglomerado de municípios que, há 10 anos, representava uma das áreas mais pobres do Nordeste e de todo o País. Faz parte do semi-árido nordestino, área exposta à ocorrência cíclica das grandes estiagens, que resultam em fracassos cruciais da produção agropecuária e no desencadeamento de crises alimentares agudas. Esta zona é considerada como uma das grandes prioridades e, ao mesmo tempo, como um dos maiores desafios da Política Nacional de Segurança Alimentar e Nutricional, pela magnitude dos problemas, pelas condições e pela expressão numérica da população exposta.

Em 2006, o município tinha uma população estimada de 4729 habitantes, distribuída na proporção de $28 \%$ na cidade e $72 \%$ no campo ${ }^{18}$. Entre 1996 e 2000, a taxa anual de crescimento demográfico foi de 1,8\%. Em 1991, seu IDH era de 0,488, elevando-se, no ano 2000, para 0,527, representado pelos sub-índices educação $(0,590)$, longevidade $(0,517)$ e renda $(0,475)$.

\section{MÉTODOS}

O estudo, do tipo transversal, foi realizado entre março a junho de 2005, com uma amostra de 501 famílias em Gameleira e 458 famílias em São João do Tigre (1 528 e 1163 adolescentes e adultos, respectivamente), em conglomerados escolhidos intencionalmente, a partir de levantamentos realizados junto às Secretarias Municipais de Saúde, considerando dois critérios: risco socioeconômico e existência de crianças menores de 5 anos.

Para o cálculo da amostra levou-se em consideração uma prevalência de, aproximadamente, $60 \%$ de insegurança alimentar para o Nordeste, com base nos resultados da PNAD, $2004^{12}$, estimando um erro máximo de $5 \%$, para um nível de significância de 95\% que, acrescida de $10 \%$ para compensar possíveis perdas, resultou em uma amostra mínima de 440 famílias. Para o cálculo amostral utilizou-se o programa Statcalc do Epi Info, versão, 6.04. No presente estudo, não se considerou a dicotomia urbano/rural da população.

A avaliação da (in)segurança alimentar foi efetuada mediante a aplicação do questionário do United States Department of Agriculture (USDA) modificado pela experiência de grupos focais no Brasil (questionário EBIA) ${ }^{13}$. O instrumento consta de 15 perguntas centrais fechadas, com resposta sim ou não sobre a experiência nos últimos três meses de insuficiência alimentar em 
seus diversos níveis de intensidade, que vão da apreensão de que a comida possa vir a faltar até a vivência de passar todo um dia sem comer. Cada resposta afirmativa do questionário equivale a um ponto, variando a contagem de 0 a 15 pontos, considerando o valor zero como a condição de segurança; 1-5 pontos como insegurança leve; 6-10 pontos como insegurança moderada e 11-15 pontos como insegurança grave. Cada pergunta central é seguida de uma pergunta sobre a freqüência com que o evento afirmativo ocorre. Neste estudo essas freqüências não foram contabilizadas.

A avaliação antropométrica foi realizada segundo procedimentos técnicos recomendados pela Organização Mundial de Saúde, de $1995^{20}$. O peso foi obtido utilizando balança digital (Filizola E-150/3P, São Paulo, Brasil) com capacidade para $150 \mathrm{~kg}$ e precisão de $100 \mathrm{~g}$. A altura foi aferida utilizando estadiômetro (Cardiomed, Curitiba - PR) com amplitude de $200 \mathrm{~cm}$ e subdivisões de $0,1 \mathrm{~cm}$. Para garantir a precisão das mensurações, as medidas foram tomadas duas vezes para cada indivíduo, observando-se que a diferença entre as avaliações não deveria exceder $0,5 \mathrm{~cm}$. No caso de extrapolar esse limite, repetia-se a mensuração e anotavam-se as duas medições com valores mais próximos, sendo utilizada a média de cada medida para efeito de registro da variável.

Para avaliação do estado nutricional dos três grupos (adolescentes de 10 a 19 anos, e adultos de 20 a 39 anos e $\geq 40$ anos), utilizou-se o cálculo da relação peso/altura pelo Índice de Massa Corporal (IMC), obtido a partir da divisão da massa corporal em quilogramas pela estatura em metro, elevada ao quadrado $\left(\mathrm{kg} / \mathrm{m}^{2}\right)$. Foram retiradas da amostra as gestantes e as nutrizes. $\mathrm{Na}$ classificação dos resultados de IMC, foram utilizadas as recomendações da Organização Mundial de Saúde $(\mathrm{OMS})^{20}$, considerando: para adolescentes: baixo peso: percentil $<5$; peso normal: percentil $\geq 5$ e $<85$; sobrepeso: percentil $\geq 85$ e $<95$ e obesidade: percentil $\geq 95$. Para adultos: baixo peso: IMC $<18,5$; normalidade: IMC $\geq 18,5$ e $<25,0$; sobrepeso: IMC $\geq 25,0 \mathrm{~kg} / \mathrm{m}^{2}$ e $<30,0 \mathrm{~kg} / \mathrm{m}^{2}$ e obesidade: IMC $\geq 30,0 \mathrm{~kg} / \mathrm{m}^{2}$.
Os dados foram digitados em dupla entrada, com a finalidade de checar sua validação utilizando o programa estatístico Epi Info, versão 6.04 (CDC, Atlanta). Para análise da consistência interna das variáveis utilizou-se o Alpha de Cronbach com um valor mínimo aceitável de 0,85, com o auxílio do Statistical Package for Social Science (SPSS), versão 12.0.1. Nas análises da associação da (in)segurança alimentar e do estado nutricional agrupado (baixo peso/normalidade versus - vs sobrepeso/obesidade) para os diferentes grupos etários foi utilizado o teste qui-quadrado de tendência e para testar a associação entre estado nutricional e faixa etária foi utilizado o qui-quadrado. Para as análises estatísticas utilizouse o programa Epi Info, versão 6.04 (CDC, Atlanta), considerando como nível de significância estatística valores de $p<0,05$.

Os projetos foram aprovados pelo Comitê de Ética do Instituto Materno Infantil Prof. Fernando Figueira (IMIP), sob o protocolo $n^{\circ} 386$, atendendo às normas regulamentares de pesquisas envolvendo seres humanos - Resolução 196/96, do Conselho Nacional de Saúde.

\section{RESULTADOS}

O instrumento para avaliar a (in)segurança alimentar (escala EBIA) evidenciou uma consistência interna muito satisfatória, visto que o Alpha de Cronbach foi de 0,93 (Gameleira) e de 0,91 (São João do Tigre), para um mínimo aceitável de 0,85 .

Do total de famílias estudadas em cada município, aproximadamente $12,0 \%$ foram classificadas na categoria de segurança alimentar, predominando, portanto, a condição de insegurança, sendo mais prevalentes as formas moderada e grave, em Gameleira (33,5\% e 36,9\%, respectivamente) e em São João do Tigre (40,2\% e $21,6 \%$, respectivamente).

Demonstra-se na Tabela 1 que, na avaliação dos indivíduos por faixa etária, os menores percentuais de ocorrência de segurança foram 
registrados nos adolescentes das duas localidades, com freqüências praticamente iguais de 8,0\% e $8,5 \%$, respectivamente. No que se refere à insegurança, a forma considerada grave prevaleceu em 48,8\% na faixa de 10 a 19 anos em Gameleira, enquanto em São João do Tigre a maior proporção de insegurança alimentar foi na forma moderada (41,4\%), também ocorrendo em adolescentes. Nos dois municípios as distribuições de freqüência das situações de segurança/insegurança alimentar e seus graus foram significantemente associados ao grupo etário ( $p \leq 0,001)$.

Do total da amostra, $27,7 \%$ e $37,5 \%$ de adolescentes e adultos dos municípios da Gameleira e de São João do Tigre, respectivamente, não fizeram as medições antropométricas. Ao analisar comparativamente as perdas de avaliações antropométricas de adolescentes e adultos, em relação às famílias, e os resultados da avaliação da (in)segurança alimentar, verificou-se que, no caso de Gameleira, as perdas amostrais foram estatisticamente significantes nas condições de segurança e insegurança leve $(p<0,05)$. No entanto, em São João do Tigre as perdas foram homogêneas, não alterando a expressão dos resultados.

Nos dois municípios, a maior proporção de normalidade antropométrica (em torno de 85\%) verificou-se no grupo de 10 a 19 anos de idade. A ocorrência de baixo peso representou o resultado que menos variou na comparação dos diferentes grupos etários. Por outro lado, consideradas isoladas ou cumulativamente, as proporções de sobrepeso e obesidade apresentaram aumentos consistentes e progressivos com a evolução das faixas etárias. Agregadas, as duas condições representam 45,6\% dos casos em Gameleira e 58,6\% em São João do Tigre, nos adultos com 40 anos e mais. Em seu conjunto, o estado nutricional apresentou diferenças estatisticamente significantes em função dos grupos etários nas duas localidades (Tabela 2).

No grupo de adolescentes das duas localidades (Tabela 3), as maiores proporções de normalidade antropométrica (percentil $\geq 5$ e percentil <85) ocorreram na categoria insegurança leve: 90,2\% em Gameleira e 92,7\% em São João do Tigre, com freqüências percentuais mais elevadas que na condição de segurança. Em Gameleira, 8,9\% dos adolescentes de famílias classificadas em situação de insegurança alimentar grave foram identificados como casos de risco de desnutrição (percentil <5). Nas duas amostras, a freqüência de normalidade antropométrica se situou entre 84,0\% (São João do Tigre) e 86,8\% (Gameleira). Os testes estatísticos evidenciaram, nas duas loca-

Tabela 1. Proporção da (in)segurança alimentar, segundo faixas etárias dos municípios da Gameleira (PE) e São João do Tigre (PB), 2005.

\begin{tabular}{|c|c|c|c|c|c|c|c|}
\hline \multirow{2}{*}{ Especificações } & \multicolumn{2}{|c|}{ 10-19 anos } & \multicolumn{2}{|c|}{$20-39$ anos } & \multicolumn{2}{|c|}{$\geq 40$ anos } & \multirow{2}{*}{$\begin{array}{c}\text { Total } \\
\mathrm{n}\end{array}$} \\
\hline & $\mathrm{n}$ & $\%$ & $n$ & $\%$ & $n$ & $\%$ & \\
\hline \multicolumn{8}{|l|}{ Gameleira $(P E)^{a}$} \\
\hline Segurança & 40 & 8,0 & 83 & 11,5 & 41 & 13,2 & 164 \\
\hline Insegurança leve & 61 & 12,2 & 126 & 17,5 & 47 & 15,1 & 234 \\
\hline Insegurança moderada & 154 & 30,9 & 259 & 36,1 & 101 & 32,5 & 514 \\
\hline Insegurança grave & 243 & 48,8 & 251 & 34,9 & 122 & 39,2 & 616 \\
\hline Total & 498 & 100,0 & 719 & 100,0 & 311 & 100,0 & 1528 \\
\hline \multicolumn{8}{|l|}{ São João do Tigre $(P B)^{b}$} \\
\hline Segurança & 30 & 8,5 & 83 & 13,8 & 27 & 13,0 & 140 \\
\hline Insegurança leve & 57 & 16,1 & 164 & 27,2 & 42 & 20,2 & 263 \\
\hline Insegurança moderada & 146 & 41,4 & 235 & 39,1 & 75 & 36,1 & 456 \\
\hline Insegurança grave & 120 & 34,0 & 120 & 19,9 & 64 & 30,7 & 304 \\
\hline Total & 353 & 100,0 & 602 & 100,0 & 208 & 100,0 & 1163 \\
\hline
\end{tabular}

${ }^{\mathrm{a}} p<0,001 ;{ }^{\mathbf{b}} p<0,001$. 
lidades, que não ocorreu associação significante entre (in)segurança alimentar e estado de nutrição.

Em relação ao grupo de 20 a 39 anos de idade, as maiores freqüências de normalidade pelo IMC foram encontradas nas condições de insegurança moderada (Gameleira) e insegurança grave (São João do Tigre): 66,7\% e 63,4\%, respectivamente. Por outra parte, as maiores ocorrências das duas condições conjuntas (sobrepeso/ obesidade) foram assinaladas nas classificações de segurança alimentar ou insegurança leve, principalmente em São João do Tigre, aonde chegou a $57,0 \%$ das amostras analisadas. Não foi encontrada associação estatística entre o estado de nutrição e a classificação da insegurança alimentar em Gameleira. No entanto, observou-se associação estatística $(p<0,001)$ nos resultados de São João do Tigre (Tabela 4).

Tabela 2. Classificação nutricional, segundo o índice de massa corporal de adolescentes e adultos, dos Municípios da Gameleira (PE) e de São João do Tigre (PB), 2005.

\begin{tabular}{|c|c|c|c|c|c|c|c|}
\hline \multirow{2}{*}{ IMC } & \multicolumn{2}{|c|}{ 10-19 anos } & \multicolumn{2}{|c|}{$20-39$ anos } & \multicolumn{2}{|c|}{$\geq 40$ anos } & \multirow{2}{*}{$\begin{array}{c}\text { Total } \\
n\end{array}$} \\
\hline & $n$ & $\%$ & $n$ & $\%$ & $n$ & $\%$ & \\
\hline \multicolumn{8}{|c|}{ Gameleira $(P E)^{a}$} \\
\hline Baixo peso & 29 & 7,3 & 17 & 3,4 & 6 & 2,8 & 52 \\
\hline Normalidade & 343 & 86,8 & 319 & 64,2 & 110 & 51,6 & 772 \\
\hline Sobrepeso & 16 & 4,1 & 110 & 22,1 & 62 & 29,2 & 188 \\
\hline Obesidade & 7 & 1,8 & 51 & 10,3 & 35 & 16,4 & 93 \\
\hline Total & 395 & 100,0 & 497 & 100,0 & 213 & 100,0 & 1105 \\
\hline \multicolumn{8}{|c|}{ São João do Tigre $(P B)^{b}$} \\
\hline Baixo peso & 9 & 3,6 & 12 & 3,4 & 2 & 1,6 & 23 \\
\hline Normalidade & 210 & 84,0 & 185 & 52,3 & 49 & 39,8 & 444 \\
\hline Sobrepeso & 25 & 10,0 & 125 & 35,3 & 58 & 47,2 & 208 \\
\hline Obesidade & 6 & 2,4 & 32 & 9,0 & 14 & 11,4 & 52 \\
\hline Total & 250 & 100,0 & 354 & 100,0 & 123 & 100,0 & 727 \\
\hline
\end{tabular}

a $p<0,001 ;{ }^{b} p<0,001$.

Tabela 3. Relação entre categorias de (in)segurança alimentar e estado nutricional de adolescentes dos municípios da Gameleira (PE) e São João do Tigre (PB), 2005

\begin{tabular}{|c|c|c|c|c|c|c|c|}
\hline \multirow{3}{*}{ (In)Segurança alimentar } & \multicolumn{7}{|c|}{ Percentis } \\
\hline & \multicolumn{2}{|c|}{$<5$} & \multicolumn{2}{|c|}{$\geq 5 \mathrm{a}<85$} & \multicolumn{2}{|c|}{$\geq 85$} & \multirow{2}{*}{$\frac{\text { Total }}{\mathrm{n}}$} \\
\hline & $\mathrm{n}$ & $\%$ & $\mathrm{n}$ & $\%$ & $\mathrm{n}$ & $\%$ & \\
\hline \multicolumn{8}{|l|}{ Gameleira $(P E)^{a}$} \\
\hline Segurança & 2 & 6,7 & 25 & 83,3 & 3 & 10,0 & 30 \\
\hline Insegurança leve & 2 & 3,9 & 46 & 90,2 & 3 & 5,9 & 51 \\
\hline Insegurança moderada & 8 & 6,6 & 107 & 87,7 & 7 & 5,7 & 122 \\
\hline Insegurança grave & 17 & 8,9 & 165 & 85,9 & 10 & 5,2 & 192 \\
\hline Total & 29 & 7,3 & 343 & 86,8 & 23 & 5,9 & 395 \\
\hline \multicolumn{8}{|l|}{ São João do Tigre $(P B)^{b}$} \\
\hline Segurança & 0 & 0,0 & 17 & 89,5 & 2 & 10,5 & 19 \\
\hline Insegurança leve & 0 & 0,0 & 38 & 92,7 & 3 & 7,3 & 41 \\
\hline Insegurança moderada & 6 & 5,6 & 83 & 77,6 & 18 & 16,8 & 107 \\
\hline Insegurança grave & 3 & 3,6 & 72 & 86,8 & 8 & 9,6 & 83 \\
\hline Total & 9 & 3,6 & 210 & 84,0 & 31 & 12,4 & 250 \\
\hline
\end{tabular}

$\chi^{2}$ de tendência linear ( $p<85$ vs $\left.p \geq 85\right){ }^{\mathbf{a}} \quad p=0,40$ e ${ }^{\mathbf{b}} \quad p=0,90$. 
No que concerne às pessoas com 40 anos e mais, enquadradas na categoria de segurança alimentar, a condição nutricional de maior ocorrência foi o binômio sobrepeso/obesidade, agrupado acima do IMC $\geq 25,0$ kg/m²: 64,0\% em Gameleira e 71,4\% em São João do Tigre. Na condição de insegurança moderada e grave, nos dois municípios, o déficit da relação peso/altura, expresso em
IMC abaixo de $18,5 \mathrm{~kg} / \mathrm{m}^{2}$, variou entre 0 (zero) e 5,9\%. No caso de Gameleira, encontrou-se uma associação estatisticamente significante $(p=0,01)$ entre níveis de (in)segurança alimentar e estado nutricional dos adultos com 40 ou mais anos de idade. Esta associação não foi encontrada nos resultados de São João do Tigre (Tabela $5)$.

Tabela 4. Relação entre categorias de (in)segurança alimentar e estado nutricional de adultos de 20-39 anos dos municípios da Gameleira (PE) e São João do Tigre (PB), 2005.

\begin{tabular}{|c|c|c|c|c|c|c|c|}
\hline \multirow{3}{*}{ (In)Segurança alimentar } & \multicolumn{7}{|c|}{ Índice de massa corporal } \\
\hline & \multicolumn{2}{|c|}{$<18,5$} & \multicolumn{2}{|c|}{$\geq 18,5$ a $<25,0$} & \multicolumn{2}{|c|}{$\geq 25,0$} & \multirow{2}{*}{$\begin{array}{c}\text { Total } \\
\mathrm{n}\end{array}$} \\
\hline & $n$ & $\%$ & $n$ & $\%$ & $n$ & $\%$ & \\
\hline \multicolumn{8}{|l|}{ Gameleira (PE)a } \\
\hline Segurança & 3 & 6,1 & 28 & 57,1 & 18 & 36,8 & 49 \\
\hline Insegurança leve & 2 & 2,5 & 47 & 59,5 & 30 & 38,0 & 79 \\
\hline Insegurança moderada & 5 & 2,6 & 128 & 66,7 & 59 & 30,7 & 192 \\
\hline Insegurança grave & 7 & 4,0 & 116 & 65,5 & 54 & 30,5 & 177 \\
\hline Total & 17 & 3,4 & 319 & 64,2 & 161 & 32,4 & 497 \\
\hline \multicolumn{8}{|l|}{ São João do Tigre $(P B)^{b}$} \\
\hline Segurança & 1 & 2,0 & 20 & 40,9 & 28 & 57,1 & 49 \\
\hline Insegurança leve & 1 & 1,1 & 39 & 41,5 & 54 & 57,4 & 94 \\
\hline Insegurança moderada & 6 & 4,3 & 81 & 57,9 & 53 & 37,8 & 140 \\
\hline Insegurança grave & 4 & 5,6 & 45 & 63,4 & 22 & 31,0 & 71 \\
\hline Total & 12 & 3,4 & 185 & 52,2 & 157 & 44,4 & 354 \\
\hline
\end{tabular}

$\chi^{2}$ de tendência linear (IMC $<25,0$ vs $\left.I M C \geq 25,0\right):{ }^{a} p=0,23$ e ${ }^{b} p<0,001$.

Tabela 5. Relação entre categorias de (in)segurança alimentar e estado nutricional de adultos $\geq 40$ anos dos municípios da Gameleira (PE) e São João do Tigre (PB), 2005.

\begin{tabular}{|c|c|c|c|c|c|c|c|}
\hline \multirow{3}{*}{ (In)Segurança alimentar } & \multicolumn{7}{|c|}{ Índice de massa corporal } \\
\hline & \multicolumn{2}{|c|}{$<18,5$} & \multicolumn{2}{|c|}{$\geq 18,5$ a $<24,9$} & \multicolumn{2}{|c|}{$\geq 25,0$} & \multirow{2}{*}{$\frac{\text { Tota }}{\mathrm{n}}$} \\
\hline & $n$ & $\%$ & $\mathrm{n}$ & $\%$ & $\mathrm{n}$ & $\%$ & \\
\hline \multicolumn{8}{|l|}{ Gameleira $(P E)^{a}$} \\
\hline Segurança & 0 & 0,0 & 9 & 36,0 & 16 & 64,0 & 25 \\
\hline Insegurança leve & 1 & 3,6 & 13 & 46,4 & 14 & 50,0 & 28 \\
\hline Insegurança moderada & 0 & 0,0 & 40 & 53,3 & 35 & 46,7 & 75 \\
\hline Insegurança grave & 5 & 5,9 & 48 & 56,5 & 32 & 37,6 & 85 \\
\hline Total & 6 & 2,8 & 110 & 51,6 & 97 & 45,6 & 213 \\
\hline \multicolumn{8}{|l|}{ São João do Tigre $(P B)^{b}$} \\
\hline Segurança & 0 & 0,0 & 4 & 28,6 & 10 & 71,4 & 14 \\
\hline Insegurança leve & 1 & 4,3 & 10 & 43,5 & 12 & 52,2 & 23 \\
\hline Insegurança moderada & 0 & 0,0 & 19 & 39,6 & 29 & 60,4 & 48 \\
\hline Insegurança grave & 1 & 2,6 & 16 & 42,1 & 21 & 55,3 & 38 \\
\hline Total & 2 & 1,6 & 49 & 39,8 & 72 & 58,5 & 123 \\
\hline
\end{tabular}

$\chi^{2}$ de tendência linear (IMC $<25,0$ vs IMC $\left.\geq 25,0\right):{ }^{\mathbf{a}} p=0,01$ e ${ }^{\mathbf{b}} p=0,45$. 


\section{DIS CUSS Ã O}

Os resultados marcantemente conflitivos deste estudo põem em relevo pelo menos três aspectos cruciais da (in)segurança alimentar e nutricional: o próprio conceito de Segurança Alimentar e Nutricional em sua versão mais atual; o instrumento de sua avaliação e, por fim, a propriedade de sua aplicação nos marcos convencionais da epidemiologia centrada no enfoque quantitativo. É pertinente ressaltar o pensamento dominante até o final do século passado, expresso como consenso no simpósio em Berkeley, em 198721: sendo "a fome uma percepção subjetiva e, portanto, uma condição não mensurável diretamente", e que deveria ser estudada de forma indireta, recorrendo-se a variáveis correlacionadas ao problema. Contrapondo-se a essa postulação, Radimer et al. ${ }^{10}$ desenvolveram um protótipo de questionário, mediante demoradas entrevistas com 32 mulheres reconhecidamente pobres dos Estados Unidos, que tiveram experiências repetidas e marcantes com as privações do consumo alimentar, as estratégias pessoais para enfrentar situações críticas, modificando as cestas de compra, limitando ou substituindo itens de consumo, escalonando os sacrifícios individuais de cada membro da família a partir de si próprios, de modo a preservar ao máximo a alimentação dos filhos menores e dos adolescentes. Foi esse processo que ofereceu o suporte observacional para a concepção e a construção do método de avaliação da (in)segurança alimentar, baseado na vivência com o desafio físico, econômico e psicológico da fome, como destaca a análise de Marin-Léon et al. ${ }^{21}$.

De fato, parece paradoxal que, em comunidades reconhecidamente pobres, com Índice de Desenvolvimento Humano (IDH) dos mais baixos da Região e do próprio País, nos quais a insegurança alimentar chega a quase $90 \%$ das famílias, com predominância das formas moderadas e graves, o estado nutricional de adolescentes e adultos apareça, comparativamente, como um dos melhores do Brasil. Dois outros contrastes: entre os adolescentes dos dois municípios, em cujas famílias foram detectadas as maiores freqüências de insegurança alimentar grave e moderada, entre as três faixas etárias comparadas, a freqüência de normalidade antropométrica se torna até superior aos valores de populações consideradas como padrão para definição do próprio critério internacional de normalidade. Assim, em Gameleira e em São João do Tigre, 86,8\% e $84,0 \%$, respectivamente, dos adolescentes foram classificados como eutróficos segundo o IMC, enquanto nos modelos normativos dos padrões de referência esta condição seria de 80,0\%. Em relação aos adultos, as proporções de baixo peso (IMC $<18,5 \mathrm{~kg} / \mathrm{m}^{2}$ ) nas duas populações estudadas se situam dentro de valores previstos para países ou populações com os melhores padrões nutricionais. Coloca-se, assim, a questão: como considerar a coexistência da pobreza e da insegurança alimentar em escalas tão elevadas com patamares tão baixos de desnutrição e até com vantagens comparativas em relação aos indicadores internacionais? Ou, com resultados nos quais o que pontifica não são os déficits, mas, justamente, o contrário, configurado na ocorrência de sobrepeso/obesidade?

Antes de tudo, é oportuno refletir sobre as ponderações de Monteiro ${ }^{22}$ discutindo as diferenças entre pobreza, fome e desnutrição, conceitos freqüentemente usados como simples sinônimos. No ideograma que ilustra sua análise, desenha-se um espaço em que desnutrição e pobreza seriam conjuntos da mesma ordem de grandeza, embora não coincidentes como duas ocorrências sobrepostas. Já a fome, que poderia ser assumida como a versão leiga da insegurança alimentar, ocuparia, relativamente, o menor espaço na representação gráfica do modelo. Na realidade, em cada população em cada tempo, em cada espaço geográfico e segundo cada método de avaliação, os três aspectos e suas respectivas dimensões podem apresentar notáveis diferenças entre si, ainda que, em princípio, os conceitos e suas medidas ou representações devam aparecer estatisticamente associados.

Nos resultados aqui expostos esta associação idealizada não existe. Ou melhor, nas raras 
situações em que aparece (caso dos adultos de 20 a 39 anos em São João do Tigre e do grupo de 40 anos e mais em Gameleira) a diferenciação estatística não resulta da hipótese que estaria sendo considerada (o baixo peso), mas do efeito matemático na distribuição do estado nutricional produzido pela elevada e discriminante proporção de sobrepeso/obesidade. Ou seja, na direção oposta ao conceito mais usual da insegurança alimentar em populações sob condições de vida muito precárias.

A relação entre segurança alimentar e estado nutricional das duas populações aqui avaliadas, tendo como traço comum o baixo IDH em ecossistemas físicos e econômicos bem diferentes, oferece uma oportunidade singular para analisar certos aspectos aparentemente surpreendentes da situação nutricional da região Nordeste, do próprio Brasil. Nessa perspectiva, chama-se a atenção para o fato de que, representando situações bem diferenciadas (a Zona da Mata e o chamado Sertão seco) as condições socioeconômicas, a elevadíssima ocorrência de insegurança alimentar e o estado nutricional de adolescentes e adultos sejam bem comparáveis entre estas áreas geográficas. Ademais, passam a se aproximar bastante do quadro epidemiológico referente à situação nutricional da população adulta de todo o País e, possivelmente, da situação dos adolescentes, um segmento biológico ainda pouco estudado no Brasil.

A partir de observações ecológicas ressalta-se que, mesmo sem atingir a escala de proporção dos dois municípios estudados, a freqüência de insegurança alimentar no Brasil e em suas diferentes regiões ${ }^{12}$ não se associa à prevalência de desnutrição de adultos ou de crianças 16,17,22,23. De fato, generaliza-se a observação de que a desnutrição energético-protéica (DEP), avaliada pela relação peso/altura, acha-se praticamente controlada, em termos epidemiológicos, em todo o País, e isto se aplicaria até mesmo para espaços territoriais crucialmente pobres, como no caso do presente estudo. Esta nova situação conduz a conclusões inusitadas, como assinalou Batista Filho ${ }^{24}$ ao observar que a maior ocorrência de normalidade antropométrica em adultos no Brasil, em função do IMC, se manifestava exatamente no espaço geográfico mais pobre: a zona semi-árida do Nordeste. Quadro semelhante acontece agora com os resultados do presente estudo, particularizando-se a observação de que, entre adolescentes da Gameleira e de São João do Tigre, a proporção de normalidade se torna, inclusive, superior aos valores estudados, aceitos e recomendados como padrões internacionais de referência ${ }^{25}$.

É evidente que esse registro deve ser devidamente ponderado. Trata-se de um momento peculiar do rápido processo de transição que agora se estende aos espaços geográficos e aos estratos socioeconômicos mais pobres e se desloca de forma diferenciada por segmentos biológicos de vulnerabilidade também distinta, dos adolescentes aos adultos. Assim, verifica-se que, na amostra de 40 anos e mais, a proporção de sobrepeso/obesidade se equivale $(45,6 \% \mathrm{em}$ Gameleira) ou até se sobrepõe $(58,5 \%$ em São João do Tigre) à encontrada nos adultos do Nordeste ou do Brasil. Esta situação se estende às três categorias de insegurança alimentar: leve, moderada e grave. A proporção de sobrepeso/ obesidade decai com a faixa desde os 40 anos e mais e, finalmente, se enquadra em parâmetros plenamente satisfatórios para populações saudáveis entre os adolescentes.

A singularidade da situação, destacando os resultados notavelmente favoráveis na faixa de 10 a 19 anos, mesmo em termos de padrões internacionais de normalidade, poderia ser explicada como um momento provavelmente rápido no trânsito dos perfis epidemiológicos que estão se sucedendo, nos últimos 25 anos. Entre os adolescentes, praticamente, está desaparecendo o baixo peso e ainda não se manifestou o problema do sobrepeso/obesidade: 5,9\% em Gameleira e 12,4\% em São João do Tigre, quando se toleraria, pelos critérios e pontos de corte adotados, uma ocorrência de 15,0\% em populações consideradas padrões $^{26}$. Esta situação, caracteristicamente transitória, constitui um fator importante no con- 
flito de resultados entre a avaliação da (in)segurança alimentar e a avaliação do estado nutricional de adolescentes e adultos.

É oportuno e pertinente considerar as limitações do próprio instrumento de avaliação da insegurança alimentar. Oriundos, a partir do estudo de Radimer et al..$^{10}$, de vivências relatadas e analisadas, de um grupo seleto de mulheres americanas que sofreram as marcantes adversidades da pobreza extrema em um país consumista e de economia extremamente competitiva, a escala de Cornell 10,27,28 e os instrumentos que a sucederam, incluindo a escala brasileira de insegurança alimentar, resgatam um componente subjetivo crucialmente impressionista - a vivência da fome, do desemprego, dos riscos de necessidades insatisfeitas, inclusive em relação ao acesso aos alimentos no dia a dia, nas semanas e nos meses seguintes, bem como as diferentes e traumáticas estratégias de sobrevivência nesse contexto de insegurança econômica, alimentar e emocional. Estas vivências e apreensões, impressas na formulação conceitual e metodológica do instrumento de medida de SAN, se tornariam, compreensivelmente, maiores e mais duradouras que a realidade da insegurança alimentar e, sobretudo, bem maior que sua expressão orgânica - a carência nutricional propriamente dita, em sua representação mais paradigmática, a desnutrição energético-protéica. Em outras palavras: a insegurança alimentar, no caso que está sendo aqui analisado e, provavelmente, no caso do Nordeste e até do Brasil como um todo, deve ser entendida mais na abordagem psicossocial ou antropológica do que no enfoque epidemiológico da DEP. Em relação a esse enfoque, é oportuno referir que os estudos demonstram que a deficiência de macronutrientes, representada em sua manifestação mais visível, já não constitui aspecto dominante da realidade nutricional no Brasil: a endemia da DEP foi ou está sendo rapidamente substituída pela epidemia da obesidade. E nessa nova conjuntura, a insegurança alimentar se dissocia do risco dos déficits nutricionais em escala macro, sobrando como espaço de estudo a questão dos micronutrientes, que será observada em futuros trabalhos, na mesma linha de validação da escala brasileira de insegurança alimentar.

Dois desdobramentos complementares do parágrafo anterior. É importante considerar que, nas localidades aqui estudadas, ao lado da condição estrutural de pobreza, se somam os efeitos conjunturais do desemprego em massa no período de entressafra da cana (abril/agosto) e das secas do semi-árido (setembro/janeiro, que podem se prolongar por períodos bem maiores). São momentos que criam insegurança permanente para as populações de baixa renda dos dois espaços geográficos. Isto, evidentemente, pode se refletir no estado de espírito da população, da qual cerca de $80 \%$ se acham dentro da linha de pobreza absoluta. Outro fator importante consiste em observar que, sob a cobertura do Programa Bolsa Família, se encontram mais de $75 \%$ das amostras estudadas nos dois municípios ${ }^{29}$. Considerando-se que mais 10\% seriam beneficiárias no Programa de Erradicação do Trabalho Infantil (PETI), isto resulta em uma cobertura de $75 \%$ a $90 \%$ de toda a população, valor que se compatibiliza com a freqüência de insegurança alimentar, atingindo $88 \%$ dos entrevistados. Assim, seria razoável supor que as próprias respostas ao questionário passariam a ser influenciadas pela condição de beneficiários dos programas sociais da grande maioria das famílias, de tal modo que suas declarações pudessem justificar ou legitimar os auxílios recebidos desses programas.

\section{AGRADECIMENTOS}

Ao Conselho Nacional de Desenvolvimento Científico e Tecnológico/Agronegócio pelo financiamento (Processo 502952/03-2, 502955/03-1 e 505808/04-8) e pela Bolsa de Produtividade em Pesquisa dos Professores Pedro Lira e Malaquias Batista Filho, aos Técnicos das Prefeituras das cidades da Gameleira e São João do Tigre, à equipe de campo e a todas as famílias, pelo apoio e colaboração.

\section{COLABORADORES}

J.S. OLIVEIRA, P.I.C. LIRA e I.C.L. VERAS participaram de todas as etapas: revisão bibliográfica, 
elaboração dos instrumentos, delineamento do estudo, coleta, processamento e análise de dados e redação do manuscrito. S.R. MAIA participou de todas as etapas do trabalho e coordenou o processamento dos dados. M.C.C. LEMOS e M.J. VIANA JUNIOR participaram da revisão bibliográfica, do delineamento do estudo e da redação do manuscrito. S.L.L.S. ANDRADE, F.C.L. PINTO, V.S. LEAL e M. BATISTA FILHO participaram da revisão bibliográfica, da elaboração dos instrumentos, do delineamento do estudo, do processamento e da análise de dados e da redação do manuscrito.

\section{FINANCI A MENTO}

Este estudo faz parte dos projetos: "Avaliação da situação alimentar e nutricional e seus fatores determinantes em conglomerados urbanos e rurais da zona da mata do estado de Pernambuco"; "Avaliação da situação alimentar e nutricional e seus fatores determinantes em conglomerados urbanos e rurais do semiárido do estado da Paraíba" e "Avaliação da (In)segurança alimentar em áreas de marcante instabilidade social e econômica do Nordeste". Apoio Financeiro: Ministério da Ciência e Tecnologia, Ministério do Desenvolvimento Social e Combate à Fome e Conselho Nacional de Desenvolvimento Científico e Tecnológico, a partir do Edital MCT/MESA/CNPq/CT-Agronegócio 01/2003 (processos: CNPq n 502952/2003-2, CNPq n 502955/2003-1 e CNPq n 505808/2004-8, respectivamente).

\section{REFERÊ NCIAS}

1. Brasil. Conselho Nacional de Segurança Alimentar. Princípios e diretrizes de uma política nacional de segurança alimentar e nutricional. Texto de referência da II Conferência Nacional de Segurança Alimentar e Nutricional. Brasília: CONSEA; 2004.

2. Brasil. Ministério das Relações Exteriores. A segurança alimentar e nutricional e o direito humano à limentação no Brasil. Brasília: IPEA; 2002.

3. Organización de las Naciones Unidas para La Agricultura y la Alimentación. Conferencia Mundial de los Alimentos. Roma: FAO; 1974.

4. Declaração de Alma-Ata. Conferência Internacional sobre Cuidados Primários de Saúde; 1978 6-12 set. Brasília: Ministério da Saúde; 2002.
5. Fundo das Nações Unidas para a Infância. Estratégia para melhorar a nutrição de crianças e mulheres nos países em desenvolvimento. New York: UNICEF; 1990.

6. Food and Agriculture Organization. Declaração de Roma sobre Segurança Alimentar Mundial e Plano de Ação da Cúpula Mundial da Alimentação. Roma: FAO; 1996.

7. World Health Organization. Diet, nutrition and the prevention of chronic diseases. Report of a Joint WHO/FAO Expert Consultation. Geneva: WHO; 2003. WHO Technical Report Series, 916.

8. Barreto SM, Pinheiro ARO, Sichieri R, Monteiro CA, Batista Filho B, Schimidt MI, et al. Análise da estratégia global para alimentação, atividade física e saúde da Organização Mundial da Saúde. Epidemiol Serv Saúde. 2005; 14(1):41-68.

9. Pérez-Escamilla R. Experiência internacional com a escala de percepção da insegurança alimentar. Cad Estud Desenv Soc Debate. 2005; 2(1):14-27.

10. Radimer KL, Olson CM, Greene JC, Campbell CC, Habicht JP. Understanding hunger and developing indicator to assess it in women and children. J Nutr Educ. 1992; 24(1 Suppl):36-44.

11. Radimer KL. Measurement of household food security in the USA and others industrialised countries. Public Health Nutr. 2002; 5(6a):859-64.

12. Instituto Brasileiro de Geografia e Estatística. Pesquisa Nacional por Amostra de Domicílios. Segurança Alimentar: 2004. Rio de Janeiro: IBGE; 2006.

13. Segall-Corrêa AM, Pérez-Escamilla R, Maranha LK, Sampaio MFA, Yuyama L, Alencar F, et al. Projeto: acompanhamento e avaliação da segurança alimentar de famílias brasileiras: validação de metodologia e de instrumento de coleta de informação: relatório técnico. Brasília: Ministério de Saúde; 2003.

14. Segall-Corrêa AM, Marin-Léon L, Helito H, PérezEscamilla R, Santos LMP, Paes-Sousa R. Transferência de renda e segurança alimentar no Brasil: análise dos dados nacionais. Rev Nutr. 2008; 21 (Suppl):39-51.

15. Mondini L, Monteiro CA. Relevância epidemiológica da desnutrição e da obesidade em distintas classes sociais: métodos de estudo e aplicação à população brasileira. Rev Bras Epidemiol. 1998; 1(1):28-39.

16. Batista Filho M, Miglioli TC. Alimentação e nutrição no nordeste do Brasil. Recife: Ed. Liceu; 2006. Série Publicações Cientificas Instituto Materno Infantil Prof. Fernando Figueira IMIP, n.12.

17. Instituto Brasileiro de Geografia e Estatística. Pesquisa de Orçamento Familiar (POF) 2002-2003. 
Análise da disponibilidade domiciliar de alimentos e do estado nutricional no Brasil. Rio de Janeiro: IBGE; 2004.

18. Instituto Brasileiro de Geografia e Estatística. População residente. 2000 [acesso 2008 abr 18]. Disponível em: <http://www.datasus.gov.br/cgi/ ibge/popmap.htm>.

19. Pinto FCL. Segurança alimentar e nutricional: o Programa Bolsa Família na realidade de um município da Zona da Mata Pernambucana [dissertação]. Recife: Universidade Federal de Pernambuco; 2007.

20. World Health Organization. Physical status: the use and interpretation of anthropometry. Geneva: WHO; 1995. Technical Report Series, 854.

21. Marín-Léon L, Segal-Corrêa AM, Panigassi G, Maranha LK, Sampaio MFA, Pérez-Escamilla R. A percepção de insegurança alimentar em famílias com idosos em Campinas, São Paulo, Brasil. Cad Saúde Pública. 2005; 21(5):1433-40.

22. Monteiro CA. A dimensão da pobreza, da desnutrição e da fome no Brasil. Estud Av. 2003; 48(17): 7-20.

23. Brasil. Ministério do Desenvolvimento Social. Chamada nutricional: um estudo sobre a situação nutricional das crianças do semi-árido brasileiro, Brasília. Cad Est Desenv Soc Debate. 2006; (4):116.
24. Batista Filho M. Sustentabilidade alimentar do semi-árido brasileiro. Recife: Ed. Liceu; 2005. Série Publicações Científicas Instituto Materno Infantil Prof. Fernando Figueira, n.11.

25. Cole TJ, Bellizzi MC, Flegal KM, Dietz WH. Establishing a standard definition for child overweight and obesity worldwide: international survey. BMJ. 2000; 320(1):1240-3.

26. De Onis M, Onyango AW, Borghi E, Siyam A, Nishida C, Siekmann J. Development of a WHO growth reference for school-aged children and adolescents. Bull World Health Org. 2007; 85: 660-7.

27. Wehler CA, Scott RR, Anderson J. The Community Childhood Hunger Identification Project: A model of domestic hunger-demonstration project in Seattle, Washington. J Nutr Educ. 1992; 24(1): 29-35.

28. Campbell CC. Food security: a nutritional outcome or a predictor variable? J Nutr. 1991; 121(1): 408-15.

29. Lira PIC, Batista Filho M. Alimentação, saúde e nutrição de conglomerados urbanos e rurais da zona da mata (PE) e do semi-árido (PB): relatórios técnicos ao CNPq. Recife: UFPE; 2006

Recebido em: 16/8/2007

Versão final reapresentada em: 25/9/2008

Aprovado em: 9/2/2009 